\title{
Parameter estimation for a model of fibronectin adsorption onto hydroxylapatite, oxidised polystyrene and nanostructured silica
}

\author{
$\begin{array}{lll}\text { T. N. Langtry } & & \text { P. Giokaris } \\ & & \text { B. K. } \text { Milthorpe }^{3}\end{array}$ \\ M. S. Lord ${ }^{4}$
}

(Received 31 October 2012; revised 24 April 2013)

\begin{abstract}
Fibronectin is a protein present in blood and the extracellular matrix which has important roles in cell adhesion and migration, wound healing and blood clotting. Three models of fibronectin adsorption, on various substrates of interest to biochemists, are compared. The first model (of Langmuir) is expressed explicitly as a time dependent function for the mass of protein adsorbed. The second model is a modification of the scaled particle theory of Reiss et al. [J. Chem. Phys., 31:369-380, 1959] and takes into account the probability of a molecule finding a sufficiently large vacant area on the adsorbing substrate surface. The third model
\end{abstract}

http://journal.austms.org.au/ojs/index.php/ANZIAMJ/article/view/6326 gives this article, (c) Austral. Mathematical Soc. 2013. Published August 16, 2013, as part of the Proceedings of the 16th Biennial Computational Techniques and Applications Conference. ISSN 1446-8735. (Print two pages per sheet of paper.) Copies of this article must not be made otherwise available on the internet; instead link directly to this URL for this article. 
extends the second model to the case in which molecules may expand the radius of their contact with the substrate upon adsorption. We used datasets obtained from experiments to compare the models. The Langmuir model is straightforward to fit to a dataset. The remaining models are fitted using a steepest descent method to minimise least squares error. We describe initial estimates for parameters for this procedure and compare the quality of fit of the models.

Subject class: 92E20

Keywords: protein adsorption, fibronectin

\section{Contents}

1 Introduction

C430

2 Models

C432

3 Data

C435

4 Identification of parameters

C435

5 Results

C438

6 Conclusions and further work

C442

References

C443

\section{Introduction}

Fibronectin is a multifunctional extracellular matrix and plasma protein that plays a major role in cell adhesion and migration, including wound healing, blood clotting and cell adhesion to biomaterial surfaces. In this article we model the process of fibronectin adsorption onto artificial materials. We 
consider three substrates: hydroxylapatite (HAp), oxidised polystyrene (PSox) and nanostructured silica $(\mathrm{Si})$.

The quantitative theory of adsorption originated in the early 20th century with Langmuir's work on gases in three dimensions in which it was postulated that the adsorption rate is proportional to the amount of free space remaining on the substrate, less any desorption [6]. The later scaled particle theory (SPT) of Reiss et al. [5, 3] used thermodynamic and geometrical arguments concerned with randomly and sequentially placing spheres that represent molecules into a volume with no overlap permitted. The SPT is valid for a sparsely populated volume but Widom [9] asserted that it is not an accurate representation of a system in equilibrium or near its jamming limit. This idea was used by Schaff and Talbot [8] in their two dimensional modelling of the interactions between particles on a surface where the particles were considered to be discs of a known radius. Van Tassel et al. [7] cited a number of experimental studies that suggested that proteins may undergo a conformational transition after adsorption, resulting in an increase in the surface contact area. Van Tassel et al. [7] and Brusatori and van Tassel [1] extended the approach used by Schaaf and Talbot [8] to incorporate the idea that particles, once adsorbed onto a random position, may spread symmetrically to a larger size at a given rate. The model presented by Brusatori and van Tassel [1] (hereafter referred to as the BvT model) is expressed as a pair of nonlinear differential equations with five parameters.

In this article we investigate the application of the BvT model to datasets obtained using a quartz crystal microbalance. In particular, we consider point estimates of the model parameters using nonlinear least squares regression. 


\section{Models}

Langmuir's model [6] is

$$
\frac{\mathrm{d} \Gamma}{\mathrm{dt}}=\mathrm{k}_{\mathrm{a}} \mathrm{c} \Gamma_{\mathrm{sat}}\left(1-\frac{\Gamma}{\Gamma_{\mathrm{sat}}}\right)-\mathrm{k}_{\mathrm{d}} \Gamma,
$$

where $k_{a}$ is the mass adsorption rate, $c$ is concentration, $\Gamma$ is the mass surface density of adsorbate, $\Gamma_{\text {sat }}$ is the maximum surface density of adsorbate and $k_{d}$ is the desorption rate.

In 1959 Reiss et al. [5] considered spheres inside a fixed volume and formulated the SPT. This was refined by later researchers [9, 8, e.g.] and adapted to a two dimensional region [8, 1, e.g.]. Adsorbing particles are represented as discs which settle at random positions on the substrate. Letting the radius of such a disk be $\alpha$, an adsorbed particle then cannot have another particle adsorb beside it whose centre would lie within $\alpha$ units of its circumference.

Brusatori and van Tassel [1] further modified the SPT model for the case in which a particle changes shape upon adsorption. Free $(\alpha)$ particles of radius $\eta_{\alpha}$, adsorbing at rate $k_{a}$, once adsorbed may spread symmetrically and instantaneously only to a larger $(\beta)$ particle of radius $\eta_{\beta}$ at a spreading rate $k_{s}$. Desorption occurs at rate $k_{d}$.

The representation of the BvT model in Figure 1 has each particle surrounded by two dashed concentric circles. The probability of a particle finding any vacant site for adsorption is denoted by $\Phi_{\alpha}$. If it lands between the smaller and larger dashed circles, then the $\alpha$ particle has insufficient room to spread and become a $\beta$ particle and so remains an $\alpha$ particle. If it lands outside all of the exclusion zones, then it will spread to become a $\beta$ particle at rate $k_{s}$. The probability associated with an adsorbed $\alpha$ particle having enough room to spread to a $\beta$ particle is denoted by $\Psi_{\alpha \beta}$. The BvT model is expressed as a pair of equations for the dimensionless densities of the two species of molecule. We denote the dimensionless densities of $\alpha$ particles and $\beta$ particles 


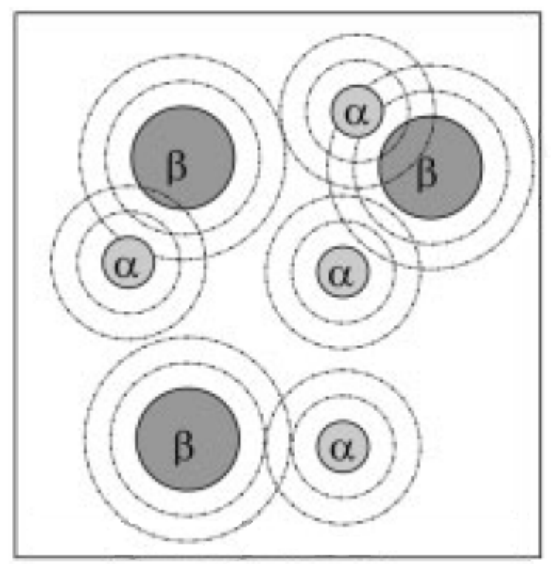

Figure 1: BvT exclusion zones. No new particle may land within the first dashed circumference surrounding each particle. Landing between the two dashed circumferences will not permit any spreading.

as $\rho_{\alpha}^{*}$ and $\rho_{\beta}^{*}$, respectively. The BvT model is then

$$
\begin{aligned}
& \frac{d \rho_{\alpha}^{*}}{d t}=k_{a} c \Phi_{\alpha}\left[t, \rho_{\alpha}^{*}(t), \rho_{\beta}^{*}(t)\right]-k_{s} \rho_{\alpha}^{*} \Psi_{\alpha \beta}\left[t, \rho_{\alpha}^{*}(t), \rho_{\beta}^{*}(t)\right]-k_{d} \rho_{\alpha}^{*}(t), \\
& \frac{d \rho_{\beta}^{*}}{d t}=k_{s} \rho_{\alpha}^{*} \Psi_{\alpha \beta}\left[t, \rho_{\alpha}^{*}(t), \rho_{\beta}^{*}(t)\right] .
\end{aligned}
$$

We denote the total dimensionless density by $\rho^{*}$, that is $\rho^{*}=\rho_{\alpha}^{*}+\rho_{\beta}^{*}$. There is an implied dependence of $\rho^{*}, \rho_{\alpha}^{*}$ and $\rho_{\beta}^{*}$ on the parameters $\eta_{\alpha}, \eta_{\beta}, k_{a}, k_{s}$, $k_{d}$ and $c$. 
Brusatori and van Tassel [1, Eq. (8-9)] give $\Phi_{\alpha}$ and $\Psi_{\alpha \beta}$ as

$$
\begin{aligned}
\Phi_{\alpha} & =(1-\tau) \exp \left[\frac{-2\left(\rho_{\alpha}^{*}+\eta \rho_{\beta}^{*}\right)}{1-\tau}-\frac{\rho_{\alpha}^{*}+\rho_{\beta}^{*}+(\eta-1)^{2} \rho_{\alpha}^{*} \rho_{\beta}^{*}}{(1-\tau)^{2}}\right], \\
\Psi_{\alpha \beta} & =\exp \left[\frac{-2(\eta-1)\left(\rho_{\alpha}^{*}+\eta \rho_{\beta}^{*}\right)}{1-\tau}-\frac{\left(\eta^{2}-1\right)\left(\rho_{\alpha}^{*}+\rho_{\beta}^{*}+(\eta-1)^{2} \rho_{\alpha}^{*} \rho_{\beta}^{*}\right)}{(1-\tau)^{2}}\right],
\end{aligned}
$$

where $\tau=\rho_{\alpha}^{*}+\eta^{2} \rho_{\beta}^{*}$ and, for simplicity, $\eta=\eta_{\beta} / \eta_{\alpha}$ (the ratio of larger radius to smaller radius). However, these equations contain errors that appear in the original article and should read

$$
\begin{aligned}
\Phi_{\alpha} & =(1-\tau) \exp \left[\frac{-2\left(\rho_{\alpha}^{*}+\eta \rho_{\beta}^{*}\right)}{1-\tau}-\frac{\rho_{\alpha}^{*}+\rho_{\beta}^{*}-(\eta-1)^{2} \rho_{\alpha}^{*} \rho_{\beta}^{*}}{(1-\tau)^{2}}\right] \\
\Psi_{\alpha \beta} & =\exp \left[\frac{-2(\eta-1)\left(\rho_{\alpha}^{*}+\eta \rho_{\beta}^{*}\right)}{1-\tau}-\frac{\left(\eta^{2}-1\right)\left(\rho_{\alpha}^{*}+\rho_{\beta}^{*}-(\eta-1)^{2} \rho_{\alpha}^{*} \rho_{\beta}^{*}\right)}{(1-\tau)^{2}}\right] .
\end{aligned}
$$

Equations (5)-(6) were used to obtain the BvT model results described in this article.

We also consider the limiting case of the BvT model in which it is assumed that just one species ( $\alpha$-type) of molecule is present. In this case we have $\Psi_{\alpha \beta}=0, k_{s}=0, \rho^{*}=\rho_{\alpha}^{*}, \eta=1, \rho_{\beta}^{*}=0$ and the adsorption model is

$$
\frac{d \rho_{\alpha}^{*}}{d t}=k_{a} c \Phi_{\alpha}\left[t, \rho_{\alpha}^{*}(t)\right]-k_{d} \rho_{\alpha}^{*}(t)
$$

where

$$
\Phi_{\alpha}\left[t, \rho_{\alpha}^{*}(t)\right]=\left(1-\rho_{\alpha}^{*}\right) \exp \left[\frac{-3 \rho_{\alpha}^{*}+2 \rho_{\alpha}^{* 2}}{\left.\left(1-\rho_{\alpha}^{*}\right)^{2}\right)}\right] .
$$

This model is related to the SPT model for fluids consisting of (three dimensional) hard spheres introduced by Reiss et al. [5] and here will be referred to as the SPT model. 


\section{Data}

Three biomaterial substrates were considered: hydroxylapatite (HAp), oxidised polystyrene (PSOx) and nanostructured silica $(\mathrm{Si})$. The data considered in this article consists of three datasets containing pairs $\left(t_{i}, d_{i}\right)$, where $t_{i}$ is the time, in seconds, at which an observation was made, and $d_{i}=d\left(t_{i}\right)$ is the corresponding observed mass density of adsorbed fibronectin measured in $\mathrm{ng} / \mathrm{cm}^{2}$. Temperature was held constant at $37^{\circ} \mathrm{C}$ and the duration of the experiments ranged from 127 to 136 minutes. Data were obtained using a quartz crystal microbalance with dissipation monitoring (D300, Q-Sense AB, Sweden) in which $30 \mathrm{~g} / \mathrm{ml}$ purified ovine fibronectin in Dulbecco's phosphate buffered saline $\mathrm{pH} 7.4$ (DPBS) was allowed to flow over the substrate material, resulting in adsorption onto the substrate.

\section{Identification of parameters}

In order to fit (2) to the experimental data we first related the dimensionless densities of the model to experimentally observed (mass) densities. We denote by $n_{\alpha}$ and $n_{\beta}$ the numbers of $\alpha$ and $\beta$ molecules, respectively, of adsorbate per $\mathrm{cm}^{2}$, and by $\mathrm{a}_{\alpha}$ the surface area of substrate occupied by an $\alpha$ particle. The total substrate areas covered by $\alpha$ and $\beta$ particles at time $t$ are then $a_{\alpha} n_{\alpha}(t)$ and $\eta^{2} a_{\alpha} n_{\beta}(t)$. We denote by $n_{\alpha \text {,sat }}$ and $n_{\beta, \text { sat }}$ the numbers of molecules per $\mathrm{cm}^{2}$ at saturation and note that $a_{\alpha}\left(n_{\alpha, \text { sat }}+\eta^{2} n_{\beta, \text { sat }}\right)$ is the total area of adsorbed $\alpha$ and $\beta$ particles at saturation. Defining $N_{\eta, s a t}=$ $n_{\alpha, \text { sat }}+\eta^{2} n_{\beta, \text { sat }}$, it follows that the fractions of saturation area (that is, the area of substrate actually covered at saturation) covered by $\alpha$ and $\beta$ particles at time $t$ are $\rho_{\alpha}^{*}$ and $\eta^{2} \rho_{\beta}^{*}$, respectively, where

$$
\rho_{\alpha}^{*}=n_{\alpha}(t) / N_{\eta, s a t}, \quad \rho_{\beta}^{*}=n_{\beta}(t) / N_{\eta, s a t} .
$$


The corresponding mass densities, in $\mathrm{ng} / \mathrm{cm}^{2}$, of $\alpha$ and $\beta$ species at $\mathrm{t}$, denoted by $\rho_{\alpha}$ and $\rho_{\beta}$, respectively, are

$$
\rho_{\alpha}(t)=m N_{\eta, s a t} \rho_{\alpha}^{*}(t), \quad \rho_{\beta}(t)=m N_{\eta, s a t} \rho_{\beta}^{*}(t) .
$$

Substituting expressions for $\rho_{\alpha}^{*}$ and $\rho_{\beta}^{*}$ derived from (8) into (2) yields

$$
\begin{aligned}
n_{\alpha}^{\prime}(t)= & c k_{a} N_{\eta, s a t} \Phi_{\alpha}\left[t, n_{\alpha}(t) / N_{\eta, s a t}, n_{\beta}(t) / N_{\eta, s a t}\right] \\
& -k_{s} n_{\alpha}(t) \Psi_{\alpha \beta}\left[t, n_{\alpha}(t) / N_{\eta, s a t}, n_{\beta}(t) / N_{\eta, s a t}\right]-k_{d} n_{\alpha}(t), \\
n_{\beta}^{\prime}(t)= & k_{s} n_{\alpha}(t) \Psi_{\alpha \beta}\left[t, n_{\alpha}(t) / N_{\eta, s a t}, n_{\beta}(t) / N_{\eta, s a t}\right]
\end{aligned}
$$

Given $\mathcal{N}$ observations of $d\left(t_{i}\right)$, we compute from (8)-(11) corresponding mass densities $\rho\left(t_{i}\right)$ of fibronectin, once the values of the parameters in those equations are identified. During experimentation the fibronectin solution was replaced by DPBS once adsorption was complete and allowed to flow over the plate with the adsorbed mass. There was no significant loss of adsorbed fibronectin for any substrate and thus we assume that the desorption rate $k_{\mathrm{d}}=0$.

The parameter identification problem was formulated as a least squares optimisation problem. In particular, given that the concentration $\mathrm{c}$ is known for each dataset and defining $n_{\text {sat }}=n_{\alpha, \text { sat }}+n_{\beta \text {,sat }}$, we seek the parameter vector $\boldsymbol{\theta}=\left(k_{s}, \eta, n_{\beta, \text { sat }}, n_{\text {sat }}, k_{a}\right)$ which minimises the sum of squared errors

$$
E(\theta)=\sum_{i=1}^{\mathcal{N}}\left[d\left(t_{i}\right)-\rho\left(t_{i} ; \theta\right)\right]^{2}
$$

subject to all entries of $\theta$ being nonnegative.

The mass $\mathrm{m}$ of a fibronectin molecule is known (approximately $4.4 \times 10^{5} \mathrm{Dal}-$ tons) a priori, as is the concentration $\mathrm{c}$ used in a particular experiment (c $=30 \mu \mathrm{g} / \mathrm{ml}$ was used in all experiments relevant to this article). Erikson and Carrell [4] reported that the molecule adopts a variety of conformations, ranging from a long thin flexible strand between 110 and $160 \mathrm{~nm}$ in length, to 
a compact helix resembling a globular protein with a Stokes radius of $8 \mathrm{~nm}$. The latter form is evident when the molecule adsorbs onto a suitable substrate [2, Fig. 3, e.g.]. In this article, the value of $\mathbf{r}_{\alpha}$ used in the SPT and BvT models was $8.0 \mathrm{~nm}$. The remaining model parameters are unknown.

The Langmuir model has an explicit solution whose form is well known, and so standard regression techniques are used to estimate the parameters of this model. However, explicit solutions are not available for the modified SPT and BvT models. Instead, a numerical solution of the governing equations was obtained using a set of initial parameter estimates, and was then used to estimate $\rho(t)$ in (12). The steepest descent method was then applied iteratively to $E$ to obtain parameter estimates that minimise $E$. For some datasets a numerical implementation of the Fletcher-Reeve method was also used, yielding similar results. The results reported in this article were all obtained by steepest descent.

The SPT model has two unknown parameters: $k_{a}$ and $n_{\text {sat }}$. Given data showing evidence of saturation, the value of $n_{\text {sat }}$ is initially approximated by $\rho\left(t_{\mathfrak{N}}\right) / \mathrm{m}$. In practice this estimate requires ad hoc modification when the data suggests that saturation has not occurred. From (7) and (9), with $\rho(0)=0$, the relation $m N_{\eta, s a t} k_{a} c=\rho^{\prime}(0)$ means that $k_{a}$ is estimated by approximating the initial slope of the data.

The BvT model requires the estimation of an additional three unknown parameters: $k_{s}, \eta$ and $n_{\beta, \text { sat }}$. We begin by nominating a feasible range for each of the parameters. Clearly we require $0 \leqslant n_{\beta, \text { sat }} \leqslant n_{\text {sat }}, 0 \leqslant k_{s}$ and $0 \leqslant \eta$. Upper bounds on feasible $k_{s}$ and $\eta$ are less obvious. In practice, small values of $k_{s}$ give rise to saturation by $\alpha$ particles while large values give rise to saturation by $\beta$ particles. To investigate reasonable search bounds for parameters we conducted multiple trial parameter identification runs using initial estimates drawn from a grid in $\mathbb{R}^{2}$ (for the SPT model) or $\mathbb{R}^{5}$ (for the BvT model), as specified in Table 1.

Given the differences in scale that apply to the components of the parameter vector $\theta$, the optimisation method was applied using the scaled variable 
Table 1: Specifications for grid of initial parameter estimates.

\begin{tabular}{cccc} 
& Lower & Upper & No. grid points \\
\hline $\log _{10} k_{\mathrm{s}}$ & -5 & 1 & 7 \\
$\eta$ & 1.01 & 12 & 12 \\
$\mathrm{n}_{\text {sat }}$ & $1.0 \times 10^{12}$ & $4.0 \times 10^{12}$ & 5 \\
$\mathrm{n}_{\beta, \text { sat }}$ & $1.0 \times 10^{9}$ & $\mathrm{n}_{\mathrm{sat}}$ & 5 \\
$\mathrm{k}_{\mathrm{a}}$ & $\mathrm{k}_{\mathrm{a}, 0} / 2$ & $\mathrm{k}_{\mathrm{a}, \mathrm{0}} \times 2$ & 3
\end{tabular}

$\hat{\boldsymbol{\theta}}=\theta_{i} /\left(10 \theta_{i, \max }\right)$, where $\theta_{i, \max }$ is the relevant upper bound given in Table 1 during each trial identification run. Also, since for the SPT and BvT models the optimisation problem (12) is defined implicitly in terms of the solution of the model equations, at some iteration $\boldsymbol{j}$ of the optimisation algorithm we estimated $\nabla E$ numerically from $\hat{\theta}_{j}$ to identify the new search direction. A linear search was then conducted in this direction with an initial step size of 0.01, applying Mathematica's inbuilt function NDSolve to (2) at each step to compute $\rho_{\alpha}$ and $\rho_{\beta}$ numerically for each new parameter vector $\hat{\theta}$ until an improving point in this direction was found, yielding the next iterate of the parameter vector $\hat{\boldsymbol{\theta}}_{j+1}$.

\section{Results}

Table 2 details the overall optimal parameter estimates obtained for each dataset and each model. It also gives the empirical relative standard deviations $\sigma_{\text {rel }}=\sigma\left(\theta_{i}\right) / \mu\left(\theta_{i}\right)$ of the distributions of individual parameter estimates for the SPT and BvT models obtained from each grid point during the optimisation procedure of Section 4.

Figure 2 shows, for the Si dataset, the best fits obtained for each of the three models and the corresponding residual plots. Corresponding plots for the HAp and PSox datasets exhibit similar qualitative characteristics. Clearly 
Table 2: Overall optimal parameter estimates $\theta_{i}$ for BvT and SPT models.

\begin{tabular}{|c|c|c|c|c|c|c|}
\hline \multirow[t]{2}{*}{ Dataset } & \multirow[t]{2}{*}{$\theta_{i}$} & \multicolumn{2}{|l|}{ BvT } & \multicolumn{2}{|c|}{$\mathrm{SPT}$} & \multirow{2}{*}{$\begin{array}{l}\text { Langmuir } \\
\text { Est. } \theta_{i}\end{array}$} \\
\hline & & Est. $\theta_{i}$ & $\sigma_{\text {rel }}$ & Est. $\theta_{i}$ & $\sigma_{\text {rel }}$ & \\
\hline \multirow[t]{5}{*}{ HAp } & $k_{s}$ & $5.3 \times 10^{-3}$ & 2.5 & & & \\
\hline & $\eta$ & 3.0 & 0.70 & & & \\
\hline & $n_{\beta, \text { sat }}$ & $1.1 \times 10^{12}$ & 1.3 & & & \\
\hline & $\mathrm{n}_{\mathrm{sat}}$ & $1.1 \times 10^{12}$ & 0.41 & $2.0 \times 10^{12}$ & $6.2 \times 10^{-3}$ & $9.5 \times 10^{11}$ \\
\hline & $k_{a}$ & $4.8 \times 10^{-9}$ & 0.64 & $3.8 \times 10^{-8}$ & $3.5 \times 10^{-2}$ & $4.5 \times 10^{-8}$ \\
\hline \multirow[t]{5}{*}{ PSox } & $k_{s}$ & 1.0 & 2.5 & & & \\
\hline & $\eta$ & 0.31 & 0.67 & & & \\
\hline & $n_{\beta, \text { sat }}$ & $3.5 \times 10^{12}$ & 1.1 & & & \\
\hline & $\mathrm{n}_{\mathrm{sat}}$ & $4.1 \times 10^{12}$ & 0.40 & $4.0 \times 10^{12}$ & $3.8 \times 10^{-3}$ & $2.0 \times 10^{12}$ \\
\hline & $k_{a}$ & $3.0 \times 10^{-7}$ & 0.66 & $4.1 \times 10^{-8}$ & $9.2 \times 10^{-3}$ & $4.0 \times 10^{-8}$ \\
\hline \multirow[t]{5}{*}{$\mathrm{Si}$} & $\mathrm{k}_{\mathrm{s}}$ & 1.5 & 2.7 & & & \\
\hline & $\eta$ & 9.2 & 0.65 & & & \\
\hline & $n_{\beta, \text { sat }}$ & $1.6 \times 10^{12}$ & 0.98 & & & \\
\hline & $\mathrm{n}_{\mathrm{sat}}$ & $4.0 \times 10^{12}$ & 0.39 & $3.5 \times 10^{12}$ & $2.7 \times 10^{-3}$ & $1.6 \times 10^{12}$ \\
\hline & $k_{a}$ & $4.7 \times 10^{-10}$ & 0.60 & $2.4 \times 10^{-8}$ & $1.4 \times 10^{-2}$ & $3.1 \times 10^{-8}$ \\
\hline
\end{tabular}

there is some autocorrelation in each set of residuals and so it is inappropriate to use the coefficient of determination in its usual form as a measure of the quality of fit of the models. However, the residual plots do suggest that the modelling errors are characterised by two components:

- a smooth component $s(t)$ that accounts for the autocorrelation;

- a stochastic component that is not autocorrelated.

Autocorrelation in residuals of deterministic nonlinear models of complex phenomena often indicates the degree to which the model fails to capture the complexity of the phenomenon. To investigate this we model the data by

$$
d_{i}=d\left(t_{i}\right)=\rho\left(t_{i} ; \theta\right)+s\left(t_{i} ; \theta\right)+\epsilon_{i} \quad \text { for } i=1, \ldots, \mathcal{N},
$$



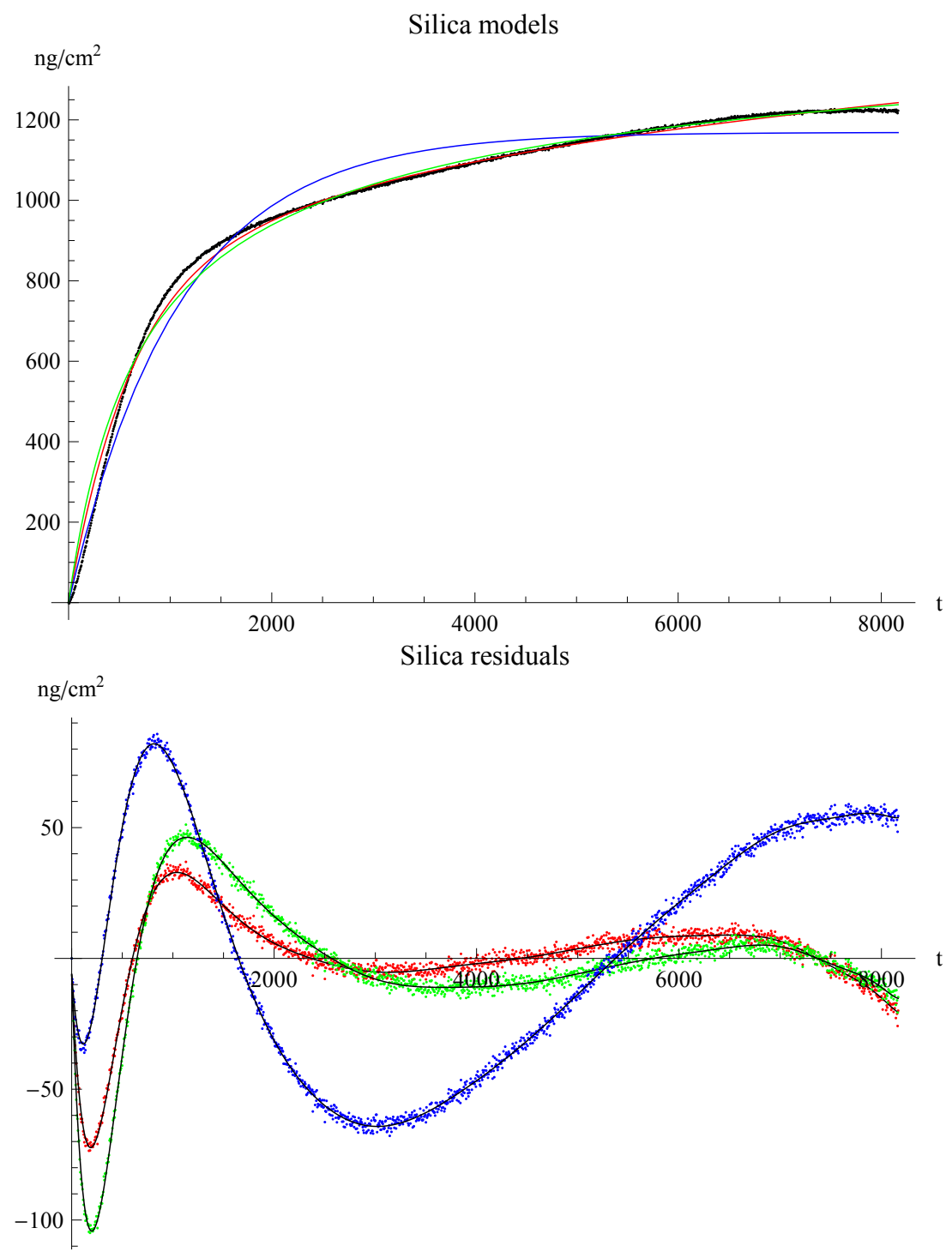

Figure 2: Top: Fitted models for the Si dataset. The data $\left(d_{i}\right)$ are plotted in black, with $\rho(t)$ in red, green and blue respectively for the BvT, SPT and Langmuir models. Bottom: Residual plots for the Si dataset. The residuals $s\left(t_{i}\right)+\epsilon_{i}$ are plotted in red, green and blue respectively for the BvT, SPT and Langmuir models. The function $s$ for each model is plotted in black. 
where $s \in C^{1}[0, \infty)$. The function $s$ was estimated in each case by fitting a trigonometric polynomial through the residuals (see Figure 2). The function $\rho+s$ then represents an estimate of the total deterministic component of the data. The Anderson-Darling test was then applied to the remaining stochastic components $\epsilon_{i}$ to test the normality of their distribution. For all datasets considered, the largest magnitude residuals occur in the early phase of observations. Values of $\epsilon_{i}$ in this early phase were also often least well described by an assumption of normality. Initial data points were deleted from the Anderson-Darling test until the $\epsilon_{i}$ corresponding to the remaining data points were iid-normally (i.e. independently and identically normally) distributed at the $p=0.05$ level. The quality of fit of a given model was then summarised by the tuple $(S, D, \mu, \sigma)$, where $S=\|s\|_{2} /\|\rho+s\|_{2}$ is a measure of the relative amount of the deterministic component not captured by the function $\rho, \mathrm{D}$ is the fraction of data points deleted from the Anderson-Darling test, and $\mu$ and $\sigma$ are the mean and standard deviation of the remaining iid-normally distributed $\epsilon_{i}$, respectively.

Table 3 details these measures for each dataset and each model. In all cases, the BvT model gives the best fit, followed by the SPT and Langmuir models, the latter providing the poorest fit for two of the three substrates. These observations suggest that the BvT model may in general be preferable as a predictive tool to the other models. However, the relative standard deviations in Table 2 indicate that, although the BvT model better accounts for the data in quantitative terms, its parameter estimates have much less precision than those of the SPT model. The parameters $k_{s}$ and $n_{\beta \text {,sat }}$ which govern the transformation of $\alpha$ particles to $\beta$ particles are particularly poorly specified. This lack of precision casts doubt on the appropriateness of the BvT model to explain the data.

The residuals, that is $s\left(t_{i}\right)+\epsilon_{i}$, obtained from the models exhibit varying degrees of autocorrelation, as seen in Figure 2. This tends to be most significant during the initial phase of adsorption, where the residuals are relatively large. In the remainder of the process residuals for the BvT and SPT models are small in magnitude relative to the data. For example, deleting the first five minutes 
Table 3: Quality of fit $S=\|s\|_{2} /\|\rho+s\|_{2}$, D is the fraction of data points deleted from the Anderson-Darling test, and $\mu_{\epsilon}$ and $\sigma_{\epsilon}$ are the mean and standard deviation of the remaining iid-normally distributed $\epsilon_{i}$.

\begin{tabular}{cclll} 
Dataset & & \multicolumn{1}{c}{ BvT } & \multicolumn{1}{c}{ SPT } & Langmuir \\
\hline HAp & S & $8.1 \times 10^{-3}$ & $2.7 \times 10^{-2}$ & $2.4 \times 10^{-2}$ \\
& $\mathrm{D}$ & 0. & 0.005 & 0. \\
& $\mu_{\epsilon}$ & $7.3 \times 10^{-4}$ & $7.2 \times 10^{-3}$ & $6.8 \times 10^{-4}$ \\
& $\sigma_{\epsilon}$ & 1.3 & 1.3 & 1.3 \\
\hline PSox & $S$ & $7.2 \times 10^{-3}$ & $2.2 \times 10^{-2}$ & $6.9 \times 10^{-2}$ \\
& $\mathrm{D}$ & 0.12 & 0.51 & 0.5 \\
& $\mu_{\epsilon}$ & $1.7 \times 10^{-2}$ & $-6.4 \times 10^{-3}$ & $-1.6 \times 10^{-2}$ \\
& $\sigma_{\epsilon}$ & 0.68 & 0.37 & 0.38 \\
\hline $\mathrm{Si}$ & $\mathrm{S}$ & $1.7 \times 10^{-2}$ & $2.4 \times 10^{-2}$ & $4.3 \times 10^{-2}$ \\
& $\mathrm{D}$ & 0. & 0. & 0. \\
& $\mu_{\epsilon}$ & $4.4 \times 10^{-4}$ & $7.1 \times 10^{-5}$ & $5.2 \times 10^{-4}$ \\
& $\sigma_{\epsilon}$ & 1.8 & 1.9 & 2.0
\end{tabular}

of data from the Si dataset yields $\max \left|\left(s\left(t_{i}\right)+\epsilon_{i}\right) / d_{i}\right| \approx 0.04$ and mean $\left|\left(s\left(t_{i}\right)+\epsilon_{i}\right) / d_{i}\right| \approx 0.007$ for the BvT model, and $\max \left|\left(s\left(t_{i}\right)+\epsilon_{i}\right) / d_{i}\right| \approx 0.15$ and mean $\left|\left(s\left(t_{i}\right)+\epsilon_{i}\right) / d_{i}\right| \approx 0.02$ for the SPT model. Corresponding results for the Langmuir model are consistently larger: for $\mathrm{Si}, \max \left|\left(s\left(t_{i}\right)+\epsilon_{i}\right) / d_{i}\right| \approx 0.17$ and mean $\left|\left(s\left(t_{i}\right)+\epsilon_{i}\right) / d_{i}\right| \approx 0.05$.

\section{Conclusions and further work}

Of the models considered, the BvT model better accounts for the observed data, although this is not surprising given its larger number of parameters. However, the lack of precision in the parameter estimates suggests that the model may be over parametrised. In particular, the existence of multiple 
near-optimal points in the parameter space suggests that the underlying assumption of the BvT model, that there are two identifiable conformations $(\alpha$ and $\beta$ ) for adsorbed particles, has limited value in explaining the data. For this reason we prefer the simpler SPT model. The SPT model usually better accounts for the data than the Langmuir model, and the precision of its parameter estimates gives some confidence that the parameters reflect stable properties of the system. While the precision of the parameter estimates for the Langmuir model are also generally good, the model usually has worse performance than the SPT model.

The relatively small magnitude of the BVT and SPT residuals after the initial phase of adsorption indicates that these models are consistent with the data in this phase. However, further work on the structure of the model is needed to account for the larger autocorrelated residuals observed in the initial phase of adsorption.

\section{References}

[1] M. A. Brusatori and P. R. van Tassel. A kinetic model of protein adsorption/surface-induced transition kinetics evaluated by the scaled particle theory. J. Colloid Interface Sci., 219(2):333-338, 1999. doi:10.1006/jcis.1999.6496 C431, C432, C434

[2] D. E. MacDonald, B. Markovic, M. Allen, P. Somasundaran, A. L. Boskey. Surface analysis of human plasma fibronectin adsorbed to commercially pure titanium materials. J. Biomed. Mater. Res., 41:120-130, 1998. http://www.ncbi.nlm.nih.gov/pubmed/9641632 $\mathrm{C} 437$

[3] E. Helfand, H. Reiss, H. L. Frisch, J. L. Lebowitz. Scaled particle theory of fluids. J. Chem. Phys., 33(5):1379-1385, 1960. doi:10.1063/1.1731417 C431 
[4] H. P. Erikson and N. A. Carrell. Fibronectin in extended and compact conformations: Electron microscopy and sedimentation analysis.

J. Biol. Chem., 258(23):14539-14544, 1983.

http://www.jbc.org/content/258/23/14539 C436

[5] H. Reiss, H. L. Frisch and J. L. Lebowitz. Statistical mechanics of rigid spheres. J. Chem. Phys., 31(2):369-380, 1959. doi:10.1063/1.1730361 C431, C432, C434

[6] I. Langmuir. The adsorption of gases on plane surfaces of glass, mica and platinum. J. Am. Chem. Soc., 40(9):1361-1403, 1918. doi:10.1021/ja02242a004 C431, C432

[7] P. R. van Tassel, L. Guemouri, J. J. Ramsden, G. Tarjus, P. Viot and J. Talbot. A particle-level model of irreversible protein adsorption with a postadsorption transition. J. Colloid Interface Sci., 207(2):317-323, 1998. doi:10.1006/jcis.1998.5781 C431

[8] P. Schaaf and J. Talbot. Surface exclusion effects in adsorption processes. J. Chem. Phys., 91(7):4401-4409, 1989. doi:10.1063/1.456768 C431, C432

[9] B. Widom. Random sequential addition of hard spheres to a volume. J. Chem. Phys., 44(10):3888-3894, 1966. doi:10.1063/1.1726548 C431, $\mathrm{C} 432$

\section{Author addresses}

1. T. N. Langtry, School of Mathematical Sciences, Faculty of Science, University of Technology, Sydney, PO Box 123 Broadway, NSW 2007, Australia

2. P. Giokaris, School of Mathematical Sciences, Faculty of Science, University of Technology, Sydney, PO Box 123 Broadway, NSW 2007, Australia 
3. B. K. Milthorpe, Faculty of Science, University of Technology, Sydney, PO Box 123 Broadway, NSW 2007, Australia

4. M. S. Lord, Graduate School of Biomedical Engineering, University of New South Wales, Sydney, NSW 2052, Australia 